\title{
Chyloabdomen in a cat with pancreatic carcinoma
}

\author{
Emilie Véran ${ }^{1, *}$, Julie Gallay-Lepoutre ${ }^{1}$, Guillaume Gory ${ }^{1}$, Pierre Guillaumot ${ }^{1}$ and Julie Duboy ${ }^{2}$ \\ ${ }^{I}$ Clinique vétérinaire Olliolis, $414 a$ Chemin des Canniers, 83190 Ollioules, France \\ ${ }^{2}$ Laboratoire Vet-Histo, 11 B Boulevard Miremonts, 13008 Marseille, France
}

\begin{abstract}
A 12-year-old spayed female domestic shorthair cat was evaluated for a 3-week history of abdominal distension. Chyloabdomen secondary to pancreatic carcinoma was diagnosed. The cat was palliatively managed using rutin and a low-fat diet. The etiology, diagnosis and management of chyloabdomen are discussed.
\end{abstract}

Keywords: Chyloabdomen, Lymphangiography, Pancreatic carcinoma.

\section{Introduction}

In human and veterinary medicine, chyloabdomen is a rare condition. It results from leakage of lipid-rich lymph into the peritoneal cavity, secondarily to obstruction or increased permeability of lymphatic vessels. In cats, it is believed to be mostly of neoplastic origin. Medical investigation relies on a systematic approach and definitive diagnosis often requires histopathological samples.

This report describes the exploration and management of a chyloabdomen secondary to pancreatic carcinoma in a 12 -year-old cat.

\section{Case Details}

A 12-year-old neutered female domestic shorthair cat was referred for evaluation of an abdominal effusion.

The owners reported an abdominal distension of three weeks duration, with conservation of general demeanor and appetite. The referring veterinarian detected an abdominal effusion. A chyloabdomen was suspected, according to the gross appearance of the liquid.

The cat was housed mainly outside. Her vaccination status was not up-to-date.

On physical examination, the cat was bright, alert and normothermic. The body condition score was of 4 out of 9 and she had an unkempt haircoat. A marked abdominal distension was noted. No pain was elicited on abdominal palpation. Cardiorespiratory parameters were within normal limits.

Abdominocentesis was performed under sedation. Approximately $300 \mathrm{~mL}$ of milky fluid were removed. Triglycerides content in the effusion was highly increased (102.84 g/L; reference range 0.30-1.60). The cytologic analysis of the fluid showed 1000 erythrocytes $/ \mu \mathrm{L}$ and 1500 leukocytes $/ \mu \mathrm{L}$, including neutrophils (48\%), monocytes (40\%) and small, mature lymphocytes (12\%). No bacteria nor neoplastic cells were visualized. Based on these findings, chyloabdomen was confirmed.
Complete blood count and biochemistry profile were within normal limits. In-house tests for feline immunodeficiency virus antibody and feline leukemia virus antigen were negative. A feline coronavirus research by polymerase chain reaction on effusion was negative. Echocardiography was unremarkable. Abdominal ultrasound showed remaining abdominal effusion and an ill-defined heterogeneous mesenteric aggregate in the cranial abdomen, assumed to be mesenteric fat reaction due to chronic effusion. No mass was identified and the pancreas showed no significant ultrasonographic abnormalities. Computed tomodensitometry (CT) with lymphangiography was planned.

Thoracic CT scan was within normal limits. On abdominal CT scan, the amorphous mesenteric aggregate, previously identified on ultrasound, was seen, surrounding the portal vein (Fig. 1).

The caudal extremity of the right lobe of the pancreas was slightly thickened and it was in contact with the mesenteric aggregate. It had ill-defined heterogenous contrast enhancement (Fig. 2).

CT scan lymphangiography was performed by injection of contrast media in perianal subcutaneous tissue, as previously described in dogs by Ando et al. (2012). The perianal area was clipped and surgically prepared. Using a 25-gauge needle, a warmed water-soluble contrast media (iodixanol, Visipaque ${ }^{\mathrm{TM}}$ 320, GE Healthcare SAS, Vélizy-Villacoublay, France) was injected in the subcutaneous tissue surrounding the anus at $0.6 \mathrm{~mL} / \mathrm{kg}$.

The administration site was subsequently massaged for 5 minutes. Images were obtained with a multi-detector helical CT scan at 5, 10, 15, 20, and 25 minutes after injection. Only two lymph nodes in the sacral region and one hypogastric were marked with iodixanol. The remaining lymphatic system was not correctly visualized. 


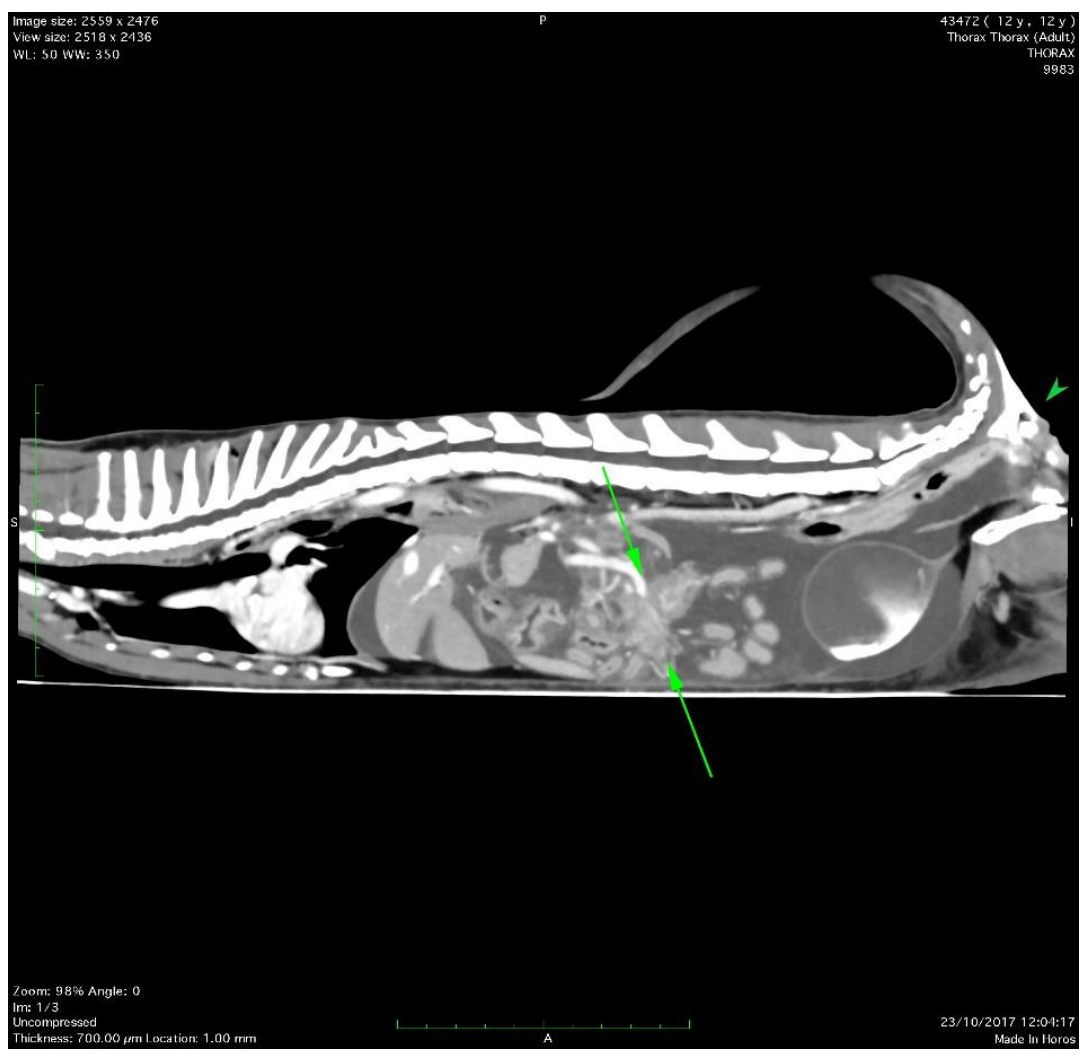

Fig. 1. Sagittal CT scan image showing a mesenteric amorphous aggregate surrounding the portal vein (arrows). The contrast media is visualized in the perianal area (arrow head) without appropriate visualization of the lymphatic system.

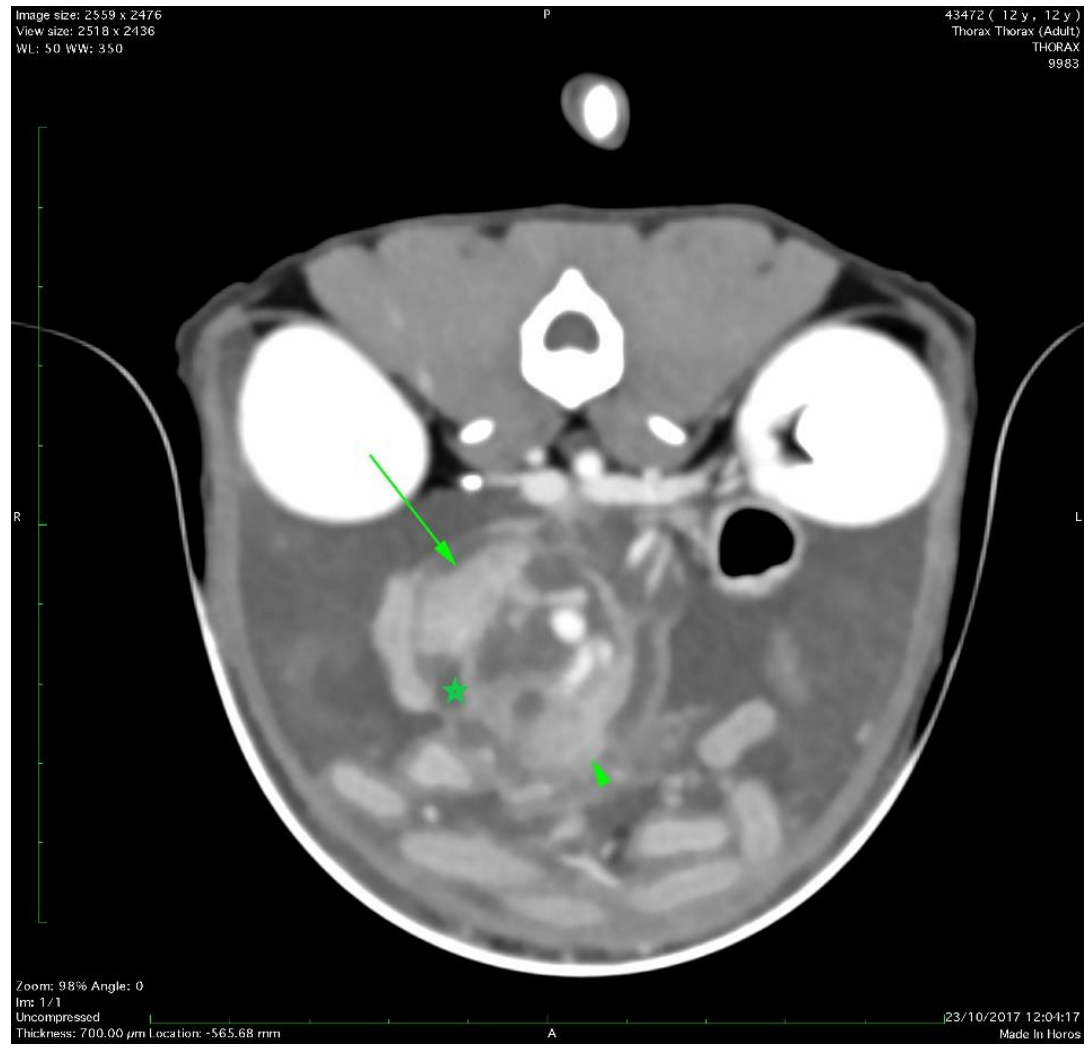

Fig. 2. Transverse CT scan image showing a contact (star) between the caudal extremity of the right lobe of the pancreas (arrow) and the mesenteric aggregate (arrow head). 
As no definitive diagnosis had been made, and given the mesenteric lesion of undetermined origin, an exploratory laparotomy was performed.

Diazepam (Valium $®$ Roche $10 \mathrm{mg} / 2 \mathrm{~mL}$, Roche, Boulogne-Billancourt, France), $0.3 \mathrm{mg} / \mathrm{kg}$ body weight (BW), IV, was given as premedication. Anesthesia was induced using alfaxalone titrated to effect (Alfaxan ${ }^{\circledR}$ $10 \mathrm{mg} / \mathrm{mL}$, Dechra Veterinary Products SAS, Montigny-le-Bretonneux, France), $3 \mathrm{mg} / \mathrm{kg} \mathrm{BW,} \mathrm{IV.}$ Oro-tracheal intubation was readily performed with a 3-mm cuffed tube.

Anesthesia was maintained with isoflurane (Vetflurane ${ }^{\circledR}$ 1000mg/g, Virbac, Carros, France) vaporized in oxygen. Analgesia was provided by morphine (Morphine Lavoisier $10 \mathrm{mg} / \mathrm{mL}$, C.D.M. Lavoisier, Paris, France), $0.2 \mathrm{mg} / \mathrm{kg} \mathrm{BW,} \mathrm{IV,} \mathrm{q2h.} \mathrm{A}$ midline laparotomy was performed.

A large amount of chylous effusion was removed. The mesentery was folded upon itself by necrotic adhesions. Those were released and biopsies of the abnormal mesentery close to the pancreas and of mesenteric and pancreaticoduodenal lymph nodes were obtained. No other abnormalities were seen on thorough examination of abdominal cavity. Abdomen wall was closed routinely.

The cat recovered well from the anesthesia. She was discharged 2 days after surgery with amoxicillin/clavulanic acid, $20 \mathrm{mg} / \mathrm{kg} \mathrm{BW}, \mathrm{PO}, \mathrm{q} 12 \mathrm{~h}$ for 5 days (Késium ${ }^{\circledR} 62.5 \mathrm{mg}$, Ceva Santé Animale, Libourne, France), rutin, $62.5 \mathrm{mg} / \mathrm{kg} \mathrm{BW}, \mathrm{PO}$, q8h, and a low-fat diet (Royal Canin gastro-intestinal low fat, Royal Canin SAS, Aimargues, France).

Biopsies revealed exocrine pancreatic tissue infiltrated by solid sheets of large polygonal cells with oval nuclei, prominent nucleoli and coarse chromatin (Fig. 3). Anisokaryosis was moderate and mitotic rate was low. Mesenteric fat tissue showed necrotic areas surrounded by vacuolated macrophages (Fig. 4). Lymph node biopsies were free from metastatic lesions. Pancreatic carcinoma with mesenteric necrotic and granulomatous remodeling was diagnosed.

Standard chemotherapy and targeted therapy (tyrosine kinase inhibitor) were declined. The cat was palliatively maintained under rutin and low-fat diet (Royal Canin gastro-intestinal low fat, Royal Canin SAS, Aimargues, France).

Three days after discharge, the cat was presented to the referring veterinarian for inappetence and depression. Non-steroidal anti-inflammatory drugs (Metacam ${ }^{\circledR} 0,5$ $\mathrm{mg} / \mathrm{ml}$ Suspension orale pour chats, Boehringer Ingelheim France Division Santé Animale, Reims, France) were prescribed for a few days and the cat improved.

On telephonic follow-up, three weeks after discharge, the patient was in good general condition. No relapse of the abdominal effusion was reported.

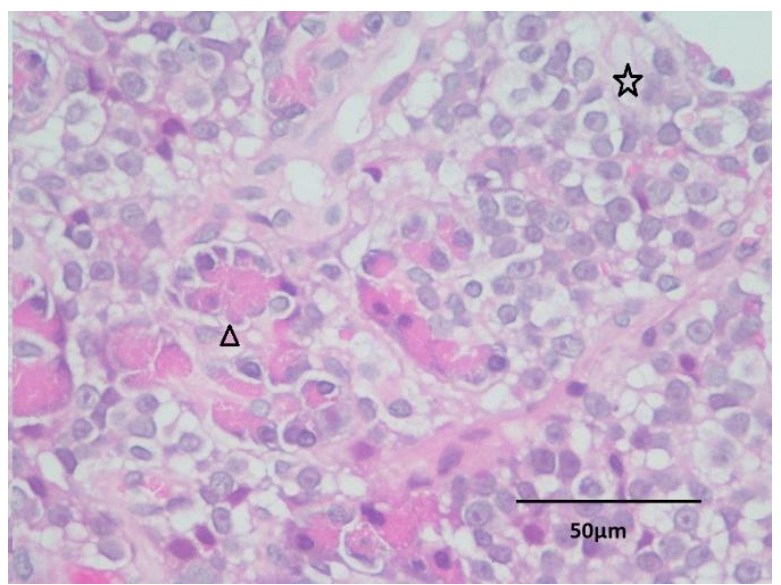

Fig. 3. Hematoxylin and eosin staining. Pancreatic carcinoma displaying a sheet of large polygonal neoplastic cells with moderate anisokaryosis and a low mitotic index (star) with residual pancreatic acini (arrow head).

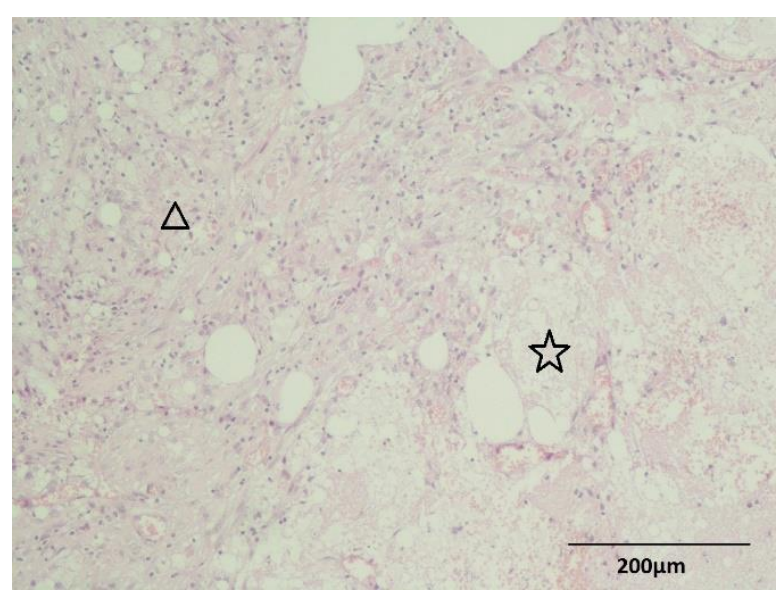

Fig. 4. Hematoxylin and eosin staining. Mesenteric fat showing necrotic area (star) surrounded by vacuolated macrophages (arrow head).

However, one month after surgery, the cat was presented to her regular veterinarian for decreased appetite. $200 \mathrm{~mL}$ of abdominal effusion were removed. Despite administration of prednisolone (Dermipred $®$ 5mg, Ceva Santé Animale, Libourne, France), maropitant (Cérénia® 16mg, Zoetis, Paris, France), and mirtazapine (Norset ${ }^{\circledR}$ 15mg, MSD France, Courbevoie, France), the cat's condition worsened with rapid recurrence of effusion. She was euthanized 52 days after the surgery.

\section{Discussion}

Chyloabdomen is an uncommon condition in veterinary medicine, with sparse data available in the literature. It results from leakage of triglycerides-rich lymph formed in intestinal lacteals into the peritoneal cavity, due to damage or obstruction of the lymphatic system or one of its tributaries (Al-Busafi et al., 2014).

Unlike chylothorax, which is frequently diagnosed as idiopathic, no idiopathic chyloabdomen has been 
described. The differential diagnosis includes tumor, congestive heart failure, infectious diseases (feline infectious peritonitis, feline immunodeficiency), ruptured cisterna chyli, mesenteric root strangulation or lymphatic vessels malformation. Cases in dogs were reported in association with intestinal lymphangiectasia (Peterson, 1996), mediastinal lymphangiosarcoma (Myers et al., 1966), acute pancreatitis (Lott et al., 2015), abdominal lymphatic rupture (Fossum et al., 1992), complication of mesenteric lymphangiography for chylothorax (Fossum et al., 1992), and lymphatic obstruction secondary to thrombus formation (Fossum et al., 1992). In contrast, chylous ascites in cats have mainly been reported in association with neoplastic disease. In the unique published case series of feline chyloabdomen, seven of 9 cats had intra-abdominal malignancy: 4 were diagnosed with a nonresectable solid tumor (hemangiosarcoma, paraganglioma), 2 with lymphoma of the small intestine infiltrating the mesenteric lymph nodes, and one with lymphangiosarcoma of the abdominal wall (Gores et al., 1994). The remaining 2 cats had nonneoplastic diseases: biliary cirrhosis, and steatitis caused by vitamin E deficiency (Gores et al., 1994). Chyloabdomen has also been associated with feline immunodeficiency virus (Börkü et al., 2005), feline infectious peritonitis (Savary et al., 2001) and hypertrophic cardiomyopathy (Nelson, 2001). A case of chylous pleural and peritoneal effusion with no underlying cause was described in a cat, with no postmortem examination performed (Thompson and Carr, 2002).

In this case report, pancreatic carcinoma was identified as the underlying cause of the chyloabdomen. Linderman et al. (2013) previously reported 34 feline cases of pancreatic carcinoma; chylous ascites was present in one cat in this series. In human medicine, direct malignant cells invasion into lymphatic vessels and obstruction of lymphatic flow by lymph node metastatic infiltration are thought to explain chyloabdomen formation during neoplastic disease (Al-Busafi et al., 2014). In this particular case, the mesenteric adipose tissue showed necrotic and granulomatous remodeling on histopathology. This might have led to an obstruction of lymphatic flow and subsequent leakage of chyle.

CT lymphangiography is an imaging technique used to assess lymphatic networks through injection of contrast agents. It is frequently used for exploration of chylous effusion when first-line diagnostic procedures have failed to identify the underlying cause of the effusion. Ultrasound-guided percutaneous lymphography by mesenteric or popliteal lymph node injection has been recently described in cats (Kim et al., 2011; Lee et al., 2012). However, the mobility of the lymph nodes and the volume of contrast agent to administer $(1.5 \mathrm{~mL})$ can make the procedure tedious (Kim et al., 2011; Lee et al., 2012). Laparotomy or more recently laparoscopy may be needed to perform the mesenteric injection (Brisson et al., 2006). In this case report, an approach described by Ando et al. (2012) was applied. Ando et al. (2012) reported an appropriate visualization of the thoracic duct 5 minutes after contrast media injection in a healthy Beagle dog. Iwanaga et al. (2016) successfully used this protocol in a Shiba Inu suffering from thoracic duct rupture, with visualization of the duct 10 minutes after injection. In this cat, however, no interpretable visualization of lymphatic system was obtained, with only a few caudal lymph nodes detected. Failure of the lymphangiography in this case might be explained by species differences in absorption of subcutaneously injected contrast media, inappropriate dose of iodixanol, a different contrast media (iopamidol in the previous descriptions) or differences in the underlying disease responsible for chylous effusion. In human medicine, the precision of magnetic resonance lymphangiography images is improved if subjects have ingested a high fat meal $3-4 \mathrm{~h}$ prior to examination rather than fasting (Chen et al., 2017). It could have been a way to enhance lymphatic networks visualization in our case.

Management of chyloabdomen first relies on treatment of the underlying cause when possible, as in this case pancreatic carcinoma.

Pancreatic carcinoma is an uncommon tumor in cats with a high metastatic rate and a poor prognosis (Linderman et al., 2013). Abdominal effusion is a negative prognostic indicator, with a median survival time of only 30 days (Linderman et al., 2013). Gemcitabine is a nucleotide analogue used as a firstline agent in human pancreatic carcinoma (Teague et $a l ., 2015)$. It has been evaluated in cats alone or in combination with other drugs, like carboplatin or tyrosine kinase inhibitors (Martinez-Ruzafa et al., 2009; Linderman et al., 2013). Chemotherapy provided an improvement of quality of life, but survival time remained poor, with a median of 165 days (Linderman et al., 2013). Only four cats were reported to live longer than a year after diagnosis; all of them received gemcitabine-based chemotherapy (Martinez-Ruzafa et al., 2009; Linderman et al., 2013). In our case, chemotherapy was declined, due to poor long-term prognosis.

A palliative treatment with rutin and a low-fat diet was instaured. A low-fat diet may decrease the amount of fat in the effusion, which may improve the animal's ability to resorb fluid from the cavity (Hawkins and Fossum, 2009).

Rutin is a benzopyrone flavonoid extracted from plants. The exact mechanism of action is unknown; it might reduce leakage from blood vessels, increase proteolysis and removal of protein from tissues, and enhance 
macrophage phagocytosis of chyle (Meadows et al., 1993; Gould, 2004; Kopko, 2005). In cats, it is recommended for management of idiopathic chylothorax (Gould, 2004; Kopko, 2005) and it was successfully used in a case of chylothorax due to cryptococcal mediastinal granuloma (Meadows et al., 1993). By analogy, rutin has been unsuccessfully used in a case of chyloabdomen secondary to hypertrophic cardiomyopathy and a chylous pleural and peritoneal effusion with no underlying condition (Nelson, 2001; Thompson and Carr, 2002). In this present case, owners have reported a great improvement of the cat general condition after initiation of rutin therapy. The hair coat was shinny and smooth, and the cat was bright and alert. However, effusion rapidly relapsed despite rutin. In conclusion, chylous ascites is an uncommon condition in dogs and cats. Neoplastic disease is a leading cause of chylous abdominal effusion in cats. The identification of the underlying cause relies on a systematic and often fastidious approach with analysis of the effusion as a first step. Regarding the lymphangiography method, the results obtained in our case were disappointing, even if the injection into the perianal tissue is easier and less invasive than into popliteal or mesenteric lymph nodes. A prospective study would be needed to validate this technique in small animal imaging and establish a standardized protocol for cats. Management of chyloabdomen relies on treatment of the underlying cause. By analogy with chylothorax, management with rutin and low-fat diet may be attempted, but to this date, no studies have demonstrated its efficacy.

\section{Conflict of interest}

The authors declare that there is no conflict of interest.

\section{References}

Al-Busafi, S.A., Ghali, P., Deschênes, M. and Wong, P. 2014. Chylous ascites: evaluation and management. ISRN Hepatology. doi.org/10.1155/2014/240473.

Ando, K., Kamijyou, K., Hatinoda, K., Shibata, S., Shida, T. and Asari, M. 2012. Computed tomography and radiographic lymphography of the thoracic duct by subcutaneous or submucosal injection. J. Vet. Med. Sci. 74, 135-140.

Börkü, M.K., Ural, K., Karakurum, M.C., Uzlu, E. and Bumin, A. 2005. Chylous pleural and peritoneal effusion in a cat with feline immunodeficiency virus; diagnosis by lipoprotein electrophoresis. Revue. Méd. Vét. 156, 612-614.

Brisson, B.A., Holmberg, D.L. and House, H. 2006. Comparison of mesenteric lymphadenography performed via surgical and laparoscopic approaches in dogs. Am. J. Vet. Res. 67, 168-173.

Chen, S., Tan, X., Wu, R., Xu, Y., Yang, C., Wang, M., Liu, F., Wang, Z. and Yua,, K. 2017. Non-enhanced MR lymphography of the thoracic duct: improved visualization following ingestion of a high fat mealinitial experience. Clin. Physiol. Funct. Imaging 37, 730-733.

Fossum, T.W., Hay, W.H., Boothe, H.W., Zack, P.M., Sherding, R.G. and Miller, M.W. 1992. Chylous ascites in three dogs. J. Am. Vet. Med. Assoc. 200, 70-76.

Gores, B.R., Berg, J., Carpenter, J.L. and Ullman, S.L. 1994. Chylous ascites in cats: nine cases (19781993). J. Am. Vet. Med. Assoc. 205, 1161-1164.

Gould, L. 2004. The medical management of idiopathic chylothorax in a domestic long-haired cat. Can. Vet. J. 45, 51-54.

Hawkins, E.C. and Fossum, T.W. 2009. Pleural effusion. In : Bonagura JD, Twedt DC, eds. Kirk's Current Veterinary Therapy XIV. Saunders Elsevier.

Iwanaga, T., Tokunaga, S. and Momoi, Y. 2016. Thoracic duct lymphography by subcutaneous contrast agent injection in a dog with chylothorax. Open Vet. J. 6, 238-241.

Kim, M., Lee, H., Lee, N., Choi, M., Kim, J., Chang, D., Choi, M. and Yoon, J. 2011. Ultrasound-guided mesenteric lymph node iohexol injection for thoracic duct computed tomographic lymphography in cats. Vet. Radiol. Ultrasound 52, 302-305.

Kopko, S.H. 2005. The use of rutin in a cat with idiopathic chylothorax. Can. Vet. J. 46, 729-731.

Lee, N., Won, S., Choi, M., Kim, J., Yi, K., Chang, D., Choi, M. and Yoon, J. 2012. CT thoracic duct lymphography in cats by popliteal lymph node iohexol injection. Vet. Radiol. Ultrasound 53, 174180 .

Linderman, M.J., Brodsky, E.M., de Lorimier, L.P., Clifford, C.A. and Post, G.S. 2013. Feline exocrine pancreatic carcinoma: a retrospective study of 34 cases. Vet. Comp. Oncol. 11, 208-218.

Lott, K., Mansfield, C. and Abraham, L.A. 2015. Acute chylous peritonitis associated with acute pancreatitis in a Staffordshire bull terrier. N. Z. Vet. J. 63, 125-126.

Martinez-Ruzafa, I., Dominguez, P.A., Dervisis, N.G., Sarbu, L., Newman, R.G., Cadile, C.D. and Kitchell, B.E. 2009. Tolerability of gemcitabine and carboplatin doublet therapy in cats with carcinomas. J. Vet. Intern. Med. 23, 570-577.

Meadows, R.L., MacWilliams, P.S., Dzata, G. and Meinen, J. 1993. Chylothorax associated with cryptococcal mediastinal granuloma in a cat. Vet. Clin. Pathol. 22, 109-116.

Myers, N.C. 3rd, Engler, S.J. and Jakowski, R.M. 1996. Chylothorax and chylous ascites in a dog with mediastinal lymphangiosarcoma. J. Am. Anim. Hosp. Assoc. 32, 263-269.

Nelson, K.L. 2001. Chyloabdomen in a mature cat. Can. Vet. J. 42, 381-383. 
Peterson, S.L.1996. Postcaval thrombosis and delayed shunt migration after pleuro-peritoneal venous shunting for concurrent chylothorax and chylous ascites in a dog. Vet. Surg. 25, 228-230.

Savary, K.C., Sellon, R.K. and Law, J.M. 2001. Chylous abdominal effusion in a cat with feline infectious peritonitis. J. Am. Anim. Hosp. Assoc. $37,35-40$.
Teague, A., Lim, K.H. and Wang-Gillam, A. 2015. Advanced pancreatic adenocarcinoma: a review of current treatment strategies and developing therapies. Ther. Adv. Med. Oncol. 7, 68-84.

Thompson, M.D. and Carr, A.P. 2002. Hyponatremia and hyperkalemia associated with chylous pleural and peritoneal effusion in a cat. Can. Vet. J. 43, 610613. 